\title{
CONF-881154--3
}

Received hy ASII

APR 061989

\section{ATOMIC PHYSICS MEASUREMENTS IN AN ELECTRON BEAM ION TRAP}

\author{
R.E. Marrs, P. Beiersdorfer, C. Bennett, M.H. Chen, T. Cowan, D. Dietrich, \\ J.R. Henderson, D.A. Knapp, A. Osterheld, M. B. Schneider, and J.H. Scofield \\ Lawrence Livermore National Laboratory
}

\author{
M.A. Levine \\ Lawrence Berkeley Laaboratory
}

This paper was prepared for submittal to

The Proceedings of the

International Symposium on $0^{-}$Beam lon Source and

Their Applications, Brookhaven National Laboratory

Upton, New York, Nevember 14-18, 1988

March 1, 1989
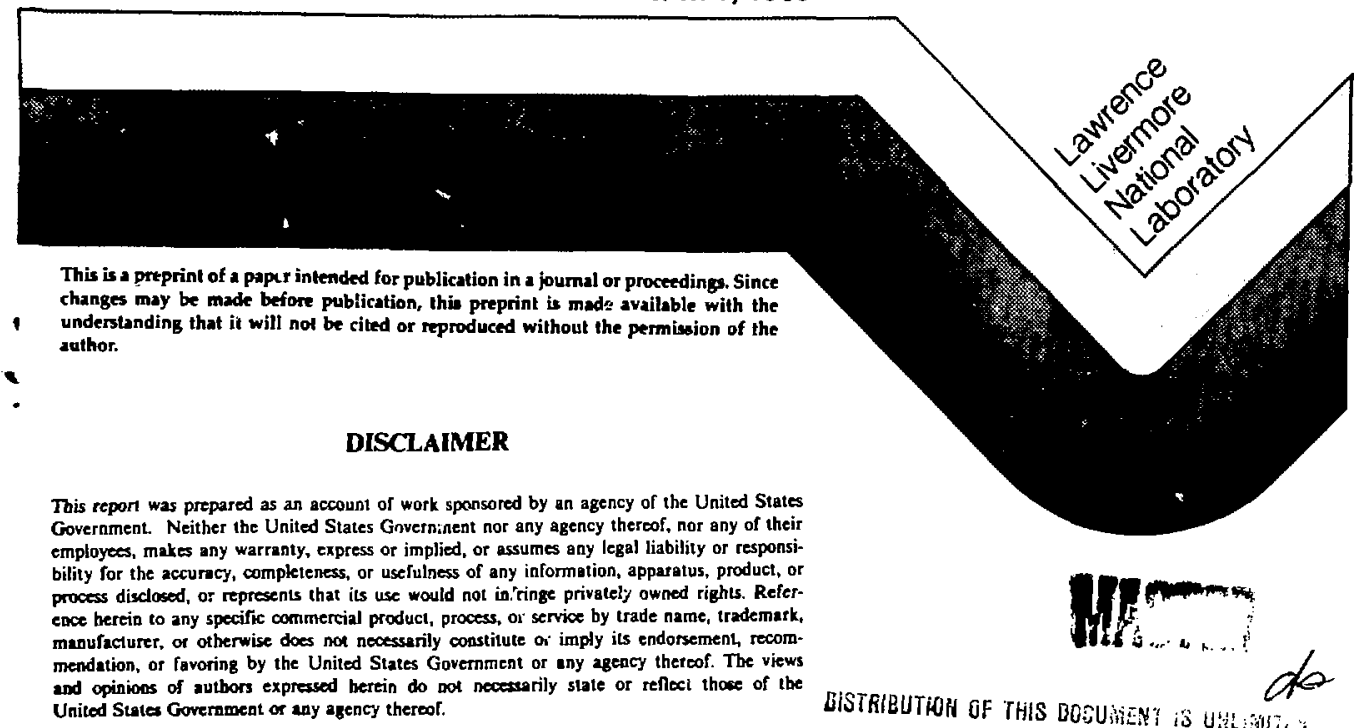
DISCLAIMER

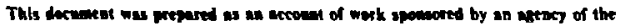

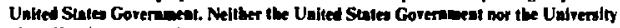

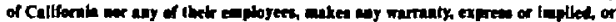

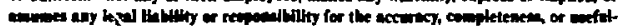

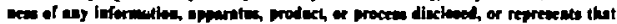

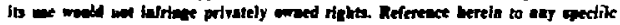

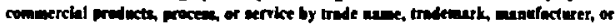

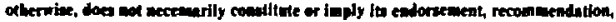
of taverias by the Uaited Sintes Goverament of the Udvenity of Californin. The

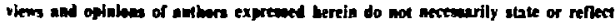

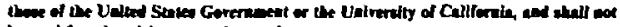

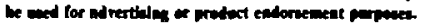




\section{ATOMIC PHYSICS MEASUREMENTS IN. AN ELECTRON BEAM ION TRAP}

R.E. Marrs, P. Beiersdorfer, C. Bennett, M.H. Chen, T. Cowan, D. Dietrich, J.R. Henderson, D.A. Knapp,

A. Osterheld. M.B. Schneider, and J.H. Scofield

Lawrence Livermore National Laboratory

UCRL-- 100725

P.O. Box 808, L-296, Livermore, CA 94550, USA

DE89 009262

M.A. Levine

Lawrence Berkeley Lalı;:atory, Berkeley, CA 94720

\section{ABSTRACT}

An electron Beam Ion Trap at Lawrence Livermore National Laboratory is being used to produce and trap very-highly-charged ions $(q \leq 70+)$ for $x$-ray spectroscopy measurements. Recent measurements of transition energies and electron excitation cross sections for $x$-ray line emission are summarized.

\section{INTRODUCTION}

A new device called an Electron Buam Ion Trap (EBIT) is operating at Lawrence Livermore National Laboratory for the purpose of studying very-highly charged ions using $x$-ray spectroscopy. The EBIT device, shown in Fig. 1, is basically a short EBIS optimized for $x$-ray spectroscopy measurements of the confined ions, for production of extremely high charge states, and for very long confinement times (up to several hours). The EBIT apparatus is described elsewhere in these proceedings by Levine.

The EBIT at LINL is being used for precision wavelength measurements of $x$-ray transitions in highly charged ions, and also for the study of electron-ion collision cross sections and excitation mechanisms for $x$-ray line emission. In all cases the EBIT device has demonstrated its ability to provide these menurements for ionization-stages which have not been accessible before. $1,2,3$ The purpose of the present paper is to provide a sampiing of these types of measurements.

\section{PRECISION WAVELENGTH MEASUREMENTS}

The small diameter of the electron beam $(70 \mu \mathrm{m})$ as it passes through the trapped ions in EBIT produces an ideal source for high resolution wavelength measurements using Bragg diffraction crystals. Two different types of spectrometers are in use. One is a Johann spectrometer, shown in Fig. 2, which can be configured for either sof $(<1 \mathrm{keV}$ ) or hard (> $3 \mathrm{keV}) \mathrm{x}$-rays. It operates in a scanning mode and uses a conventional $x$-ray tube for wavelength calibration. The other type of spectrometer uses the von Hamos geometry, in which the crystal is flat in the diffraction plane but curved out of the diffraction plane to provide [ocusing. ${ }^{4}$ A position sensitive proportional counter is used for $x$-ray detection. The wavelength calibration for this spectrometer comes from $x$-ray transitions of known energy produced in EBIT itself. 


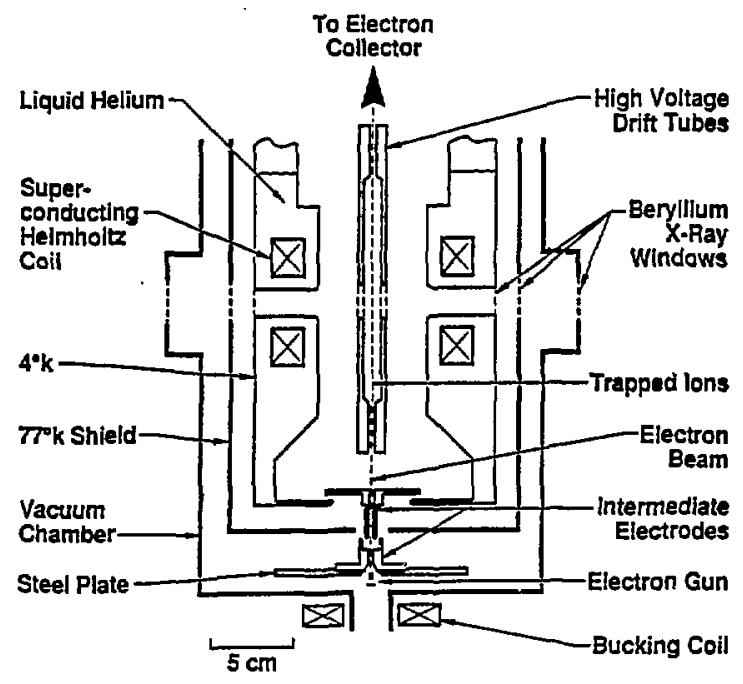

Fig 1. - The electron beam ion trap. The beryllium windows can be removed for soft $x$-ray or VUV spectroscopy. Two different drift-aube geometries have been used. In the one shown here the drift tube structure operates at a single electrical potential. In the other version (not shown), three electrically isolated drift tubes can be biased at different potentials to form a higher axial barrier.

The Johann spectrometer is being used for Lamb shift measurements in hydrogenlike and heliumlike high $-Z$ ions. These measurements are motivated by the desire to test QED predictions in the strong field limit. We have performed a preliminary experiment on $\mathrm{H}$-like nickel, in which we measured the $2 p$ to is transition energies, in order to compare the measured Lamb shift with theory. Fig. 3 displays a partial spectrum for $\mathrm{H}$-like nickel. Our preliminary measurements are in agreement with theory, and promise eventual precision much better than has been previousiy obtained from high $Z$ ion beams prcduced at accelerators.

The von Hamos spectrometer has been used to observe $x$-ray spectra from highly charged neonlike ions. For example, we have measured the $\left.\left(2 s_{1}\right)_{2} 3 p_{3 / 2}\right)_{j=1} \rightarrow$ ground state transition in Ne-like ytterbium $(Z=70)$. This transition is of interest because it allows studying the screening of the self energy in a multielectron ion. In order to determine the energy of this transition. the spectrum was calibrated by recording the position of the Lyman-alpha lines of hydrogenic zinc, as shown in Fig. 4. 


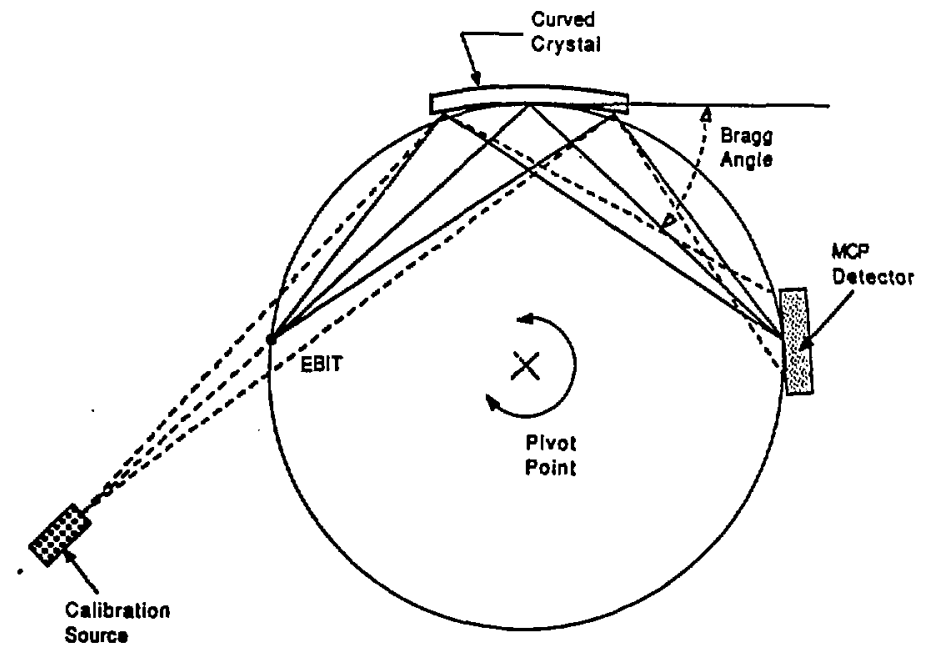

Fig. 2 - Johanm spectrometer arrangement. For soft $x$-ray measurements a position-sensitive microchannel-plate detector is located tangent to the Rowland circle, and the spectrometer operates in ultrahigh vacurm. For higher $x$-ray energies a position sensitive proportional counter is placed on the Rowland circle facing the crystal, and a vacurm chamber is unnecessary. For scanning operation the crystal and detector move together while EBII and the calibration source remain fixed.

A preliminary analysis indicates that the measured transition energy is 4 eV less than theoretical calculations. This agrees with differences foind earlier between experimental and calculated values in neonlike silver ( $Z=47$ ), $x$ enon $(Z=54)$, lanthanum $(Z=57)^{5}$, go?d $(Z=79)$ and bismuth $(Z=83) .^{6}$

\section{DIELECTRONIC RECOMBINATION MEASUREMENTS}

Dielectronic recombination (DR) is the resonant capture of an incident electron into a doubly excited state followed by $x$-ray emission:

$$
A q^{+}+e^{-} \rightarrow[A(q-1)+]^{* *} \rightarrow A(q-1)++Y D R
$$

The related processes of resonant excitation (RE) can also produce $x$-ray emission at similar energies:

$$
A q++e^{-} \rightarrow\left[A^{(q-1)+}\right]^{* *} \rightarrow\left[A^{q+}\right]^{*}+e^{-} \rightarrow A^{q+}+e^{*}+Y R E
$$




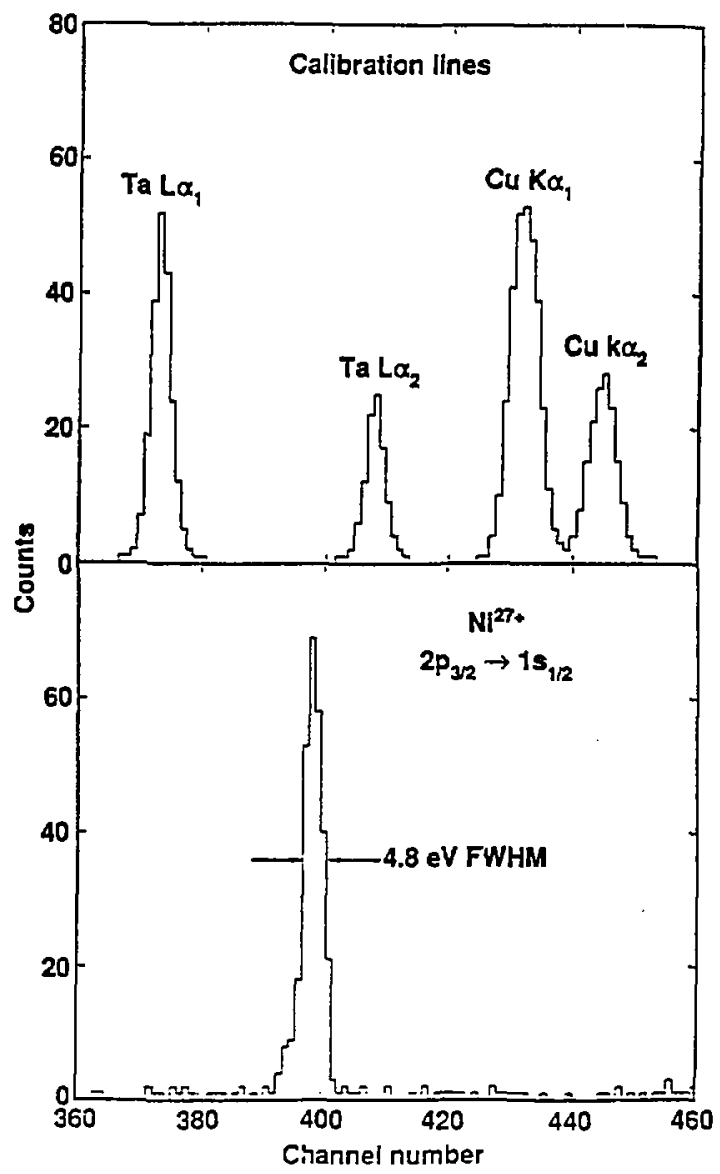

Fig. 3 - Data from the Johann spectrometer. Top: The different calibration lines shown were measured in separate scans with different exposure times. Bottom: The $2 \mathrm{p}_{3 / 2} \rightarrow 1 s_{1 / 2}$ line from $\mathrm{Ni}^{27+}$ ions in EBIT. The x-ray energy range shown is approximately $8000-8170 \mathrm{eV}$. 


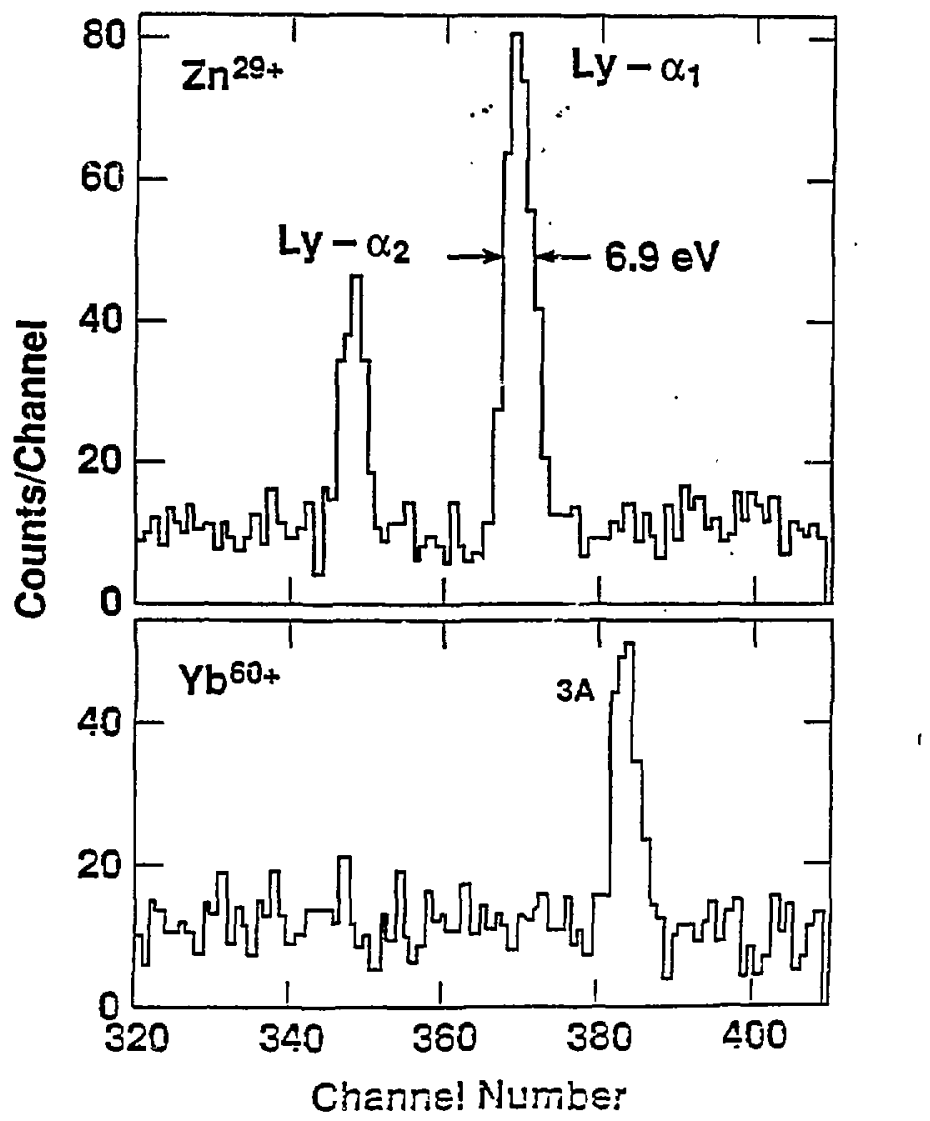

Fig. 4 - Spectra from the von Hamos spectrometer. Top: Lyman-alpha calibration lines from H-like zinc, Bottom: The $\left.(2 \leq I)_{2} 3 \mathrm{p}_{3 / 2}\right)_{\mathrm{J}=1}+\mathrm{g} . \mathrm{s}$. transition, denoted 3A, in Ne-like ytterbium. 
The DR process is important in hot plasmas, whose $x$-ray emission spectra contain satellite lines characteristic of DR. However there are no detailed measurements of DR cross sections for the high ionization stages (such as $\mathrm{He}-$ like $\mathrm{Fe}^{24+}$ or $\mathrm{Ni}^{26+}$ ) which are produced in tokamaks 7,8 and solar flares. ${ }^{9}$ Because of the precise control of the electron beam energy in EBIT and the good energy resolution, EBIT is a powerful tool for measuring a resonant process such as DR.

Since the DR cross sections being measured are quite large, setting the electron beam energy on a DR resonance quickly destroys the charge state being measured. We overcotue this problem by rapidly switching the electron energy between the resonant energy and a higher nonresonant energy which restores the ionization bulance. Typically only $\sim 10 \%$ of the time is spent on resonance. An excitation function is obtained by taking successive data runs in which the lower electron energy (i.e. the DR energy) is changed by a small amount (typically 20-eV steps) and normalizing each run to a common norresonant upper energy.

We have measured a DR excitation function for He-like $\mathrm{Ni}^{26+}$ by detecting the $\mathrm{K} \times$-rays from the recombined $\mathrm{Ni}^{25+}$ ions. For He-like target ions DR produces one and only one $K x$-ray photon; so the number of $K$ $x$-rays produced at a given incident electron energy is proportional to the $D R$ cioss section. There is one previous observation of $D R x$-rays from an EBIS. In that case an $x$-ray signal from DR of Ar ${ }^{14+}$ jons was observed. 10

Examples of EBIT spectra obtained in a Ge detector at three different electron energies are shown in Fig. 5. We have obtained cross sections for the KLL DR resonant feature by normalizing the $K x$-rays from $D R$ to theoretical radiative recombination cross sections, whose characteristic $\mathbf{x}$-rays are also clearly observed in our spectra. The results are displayed in Fig. 6 and compared to a multiconfiguration Dirac Fock calculation. 11

\section{ELECTRON IMPACT EXCITATION MEASUREMENTS}

Electron impact excitation (IE) cross sections may be obtained in a manner similar to the DR measurements described above. In this case, however, the electron energies are carefully chosen to avoid resonances, since they can influence the observed line intensities. To date, we have obtained impact excitation data for two Ne-like ions in different regions of the periodic table, $\mathrm{Ba}^{46+}$ and $\mathrm{Au}^{69+}$. This should help provide an understanding of the role of relativistic end QED effects in electron-ion collisions. Since some of the fine structure splittings in these ions are unresolved in a $\mathrm{Si}(\mathrm{Li})$ or $\mathrm{Ge}$ detector, we have also obtained spectra in a Bragg diffraction spectrometer. This spectrometer employed a flat crystal (for broad spectral coverage) and a position sensitive proportional counter.

Our $\mathrm{Ba}^{46+}$ IE cross section measurements have been published elsewhere. 1 Briefly, cross section information was obtained for five $n=3$ excited states in Ne-like barium. The results generally support existing theoretical collision strengths. 12

Electron impact excitation of Ne-like $\mathrm{Au}^{69+}$ was studied at an excitation energy of $18 \mathrm{keV}$ in the same manner as for $\mathrm{Ba}^{46+}$. Gold $\mathrm{x}-\mathrm{ray}$ spectra from a Ge detector and a Bragg diffraction crystal are shown in Figs, 7 and 8, respectively. Analysis of these data is still in progress. 


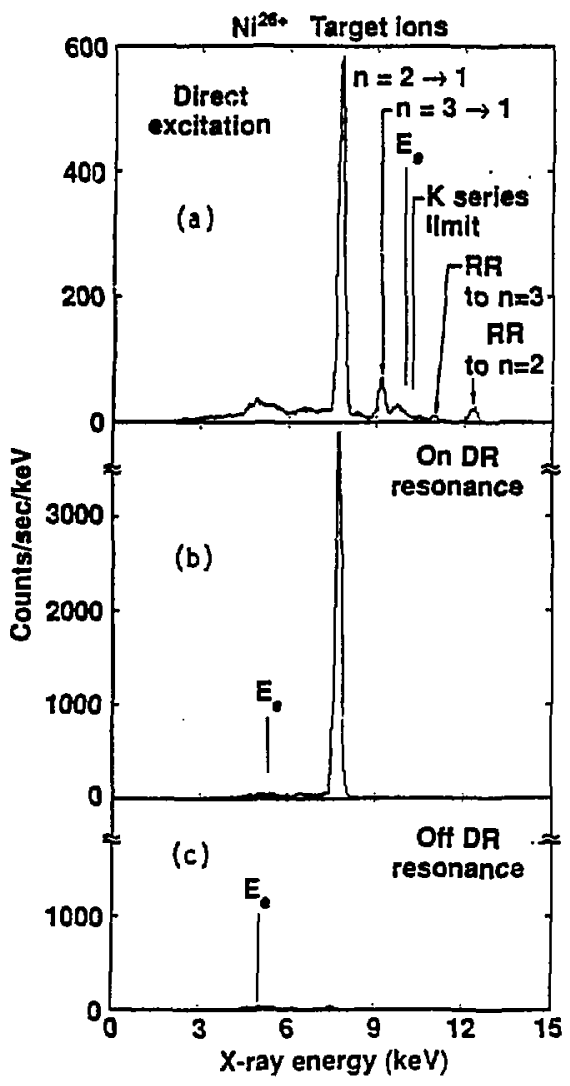

Fig. 5 - X-ray spectra from $\mathrm{Ni}^{26+}$ target ions excited at throe different electron besm energies. (a) Above the $n=2$ direct-excitation threshold, $E_{e}=$ $10.0 \mathrm{keV}$; (b) On resonance, $E_{\mathrm{e}}=5.34 \mathrm{keV}$; (c) Off resonance, $E_{\mathrm{e}}=5.05 \mathrm{keV}$. 


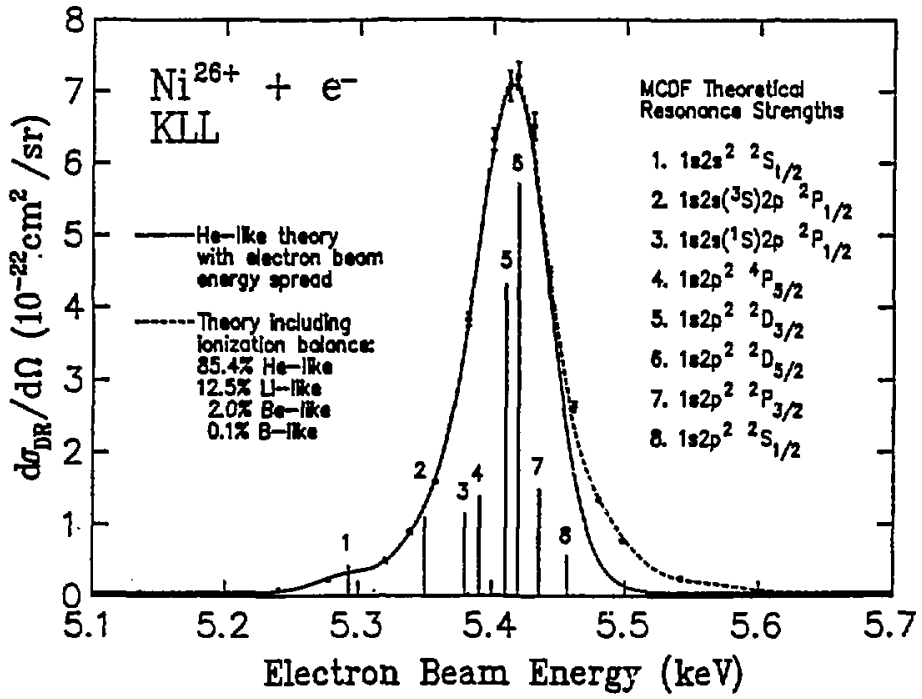

Fig. 6 - Structure of the KLL DR feature compared to theory for He-like Nz target ions. The vertical bars are the theoretical resonance strengths. The curve is the theoretical cross section obtained by folding the calculated resonance strengths with the experimental electron energy resolution function. The data points at electron energies $\gtrsim 5.4 \mathrm{keV}$ show a contribution from DR on lower-charge-state Ni ions, which are also present in the trap.

However some interesting observations can already be made. The $\left.\left(2 p_{3}\right]_{2}{ }^{3} p_{1 / 2}\right)_{j=2}$ transition shows a selative intensity stronger than expected from observations of lower $Z$ ions such as $B a^{46+}$. Model calculations predict that this line is blended with a magnetic dipole transition $\left.\left(2 \mathrm{p}_{3}\right)_{2} 3 \mathrm{p}_{1 / 2}\right)_{\mathrm{J}=1}$. The $\mathrm{Ml}$ line is absent in the spectra of ions below $\mathrm{Z} \approx 74$ due to unfavorable branching ratios. However, in the case of gold it is predicted to have an intensity which is $30 \%$ the size of the $\left(2 \mathrm{p}_{3}\right)_{2}$ $\left.3 \mathrm{p}_{1 / 2}\right)_{2}$ transition.

\section{X-RAY LINE EXCITATION MECHANISMS}

The intensity ratios of $x$-ray lines from highly charged ions are of ten used to infer the properties of the medium containing the emitting ions. 


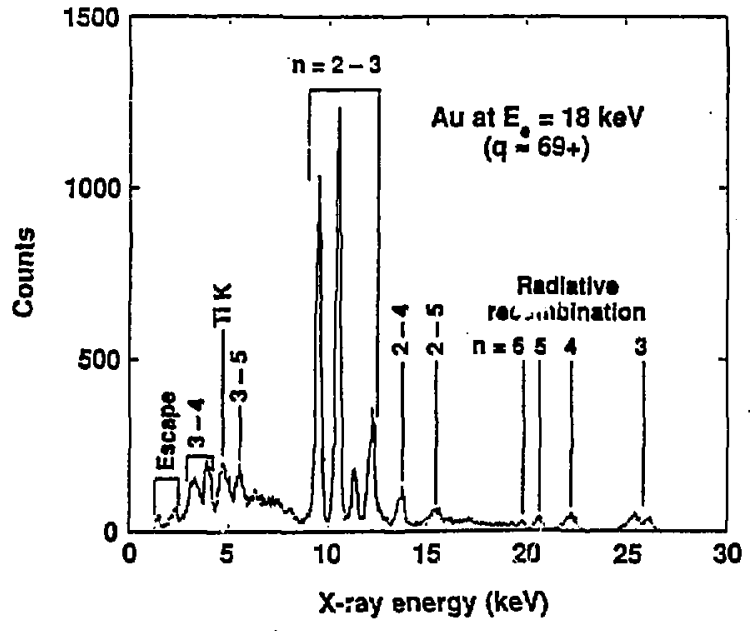

Fig. 7 - X-ray spectrum from highly ionized gold (mostly $q=69+$ and 68+) obtained in a Ge detector at an incident electron energy of $18.0 \mathrm{keV}$.

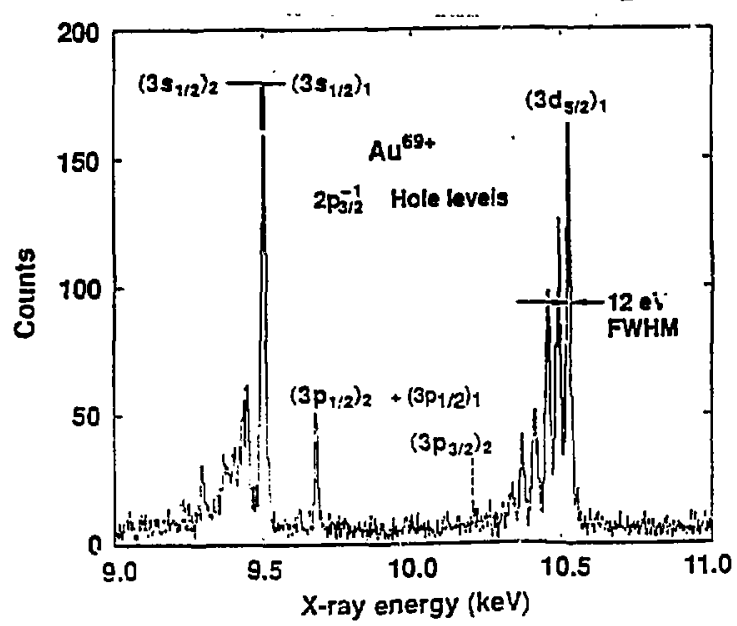

Fig. 8 - Spectrum of the 2p3/2 hole transitions in Ne-like $\mathrm{Au}^{69+}$ obrained with a flat LiF 220 Bragg diffraction crystal. The unlabeled satellite lines are transitions in $\mathrm{Na}$ - and $\mathrm{M} / \mathrm{g}$-like gold. 
This is especially true in the case of astrophysical and laser-produced plasmas where alternative methods for determining quantities such as electron density or electron temperature are unavailable. However, the interpretation of the $\mathrm{x}$-ray line emission is often model dependent, and uncertainties in the atomic models translate into uncertainties in the inferred quantities.

We have begun to investigate the intensity ratios of the $x$-ray line emission of $\mathrm{Ba}^{46+}$ as a function of electron beam enerby in order to study the effect of cascades on the $x$-ray line intensities. In Fig. 9 we have plotted the ratio of the intensity of the (2PI/2 $\left.3 s_{1 / 2}\right)_{1}$ transition (labeled $3 F$ ) and the (2p3/2 $\left.3 d_{5 / 2}\right)_{1}$ transition (nabeled 3D) vers:s beam energy. Also shown in the figure are theoretical predictions for the line ratio from a collisional-radiative model which includes all collisions and radiative dacays invoiving levels of the type $22 \mathrm{n} 2$. The collision strengths, energy levels, and radiative transition rates used in the model are from an atomic physics package developed by Klapisch et al. 13 The figure shows that a significantly higher theoretical line ratio is obtained when the number of levels in the calculation is increased. The 37-levei model, which includes the oround state and the 36 levels of the type $2 l 3 l$ ' predicts a line ratio which is $30 \%$ lower than the experimental result at $8 \mathrm{keV}$. Much better agreement with the data is found if the calculations include all 89 levels with excited electrons in the $n=3,4$ shells, or if the calculations include all 157 levels with

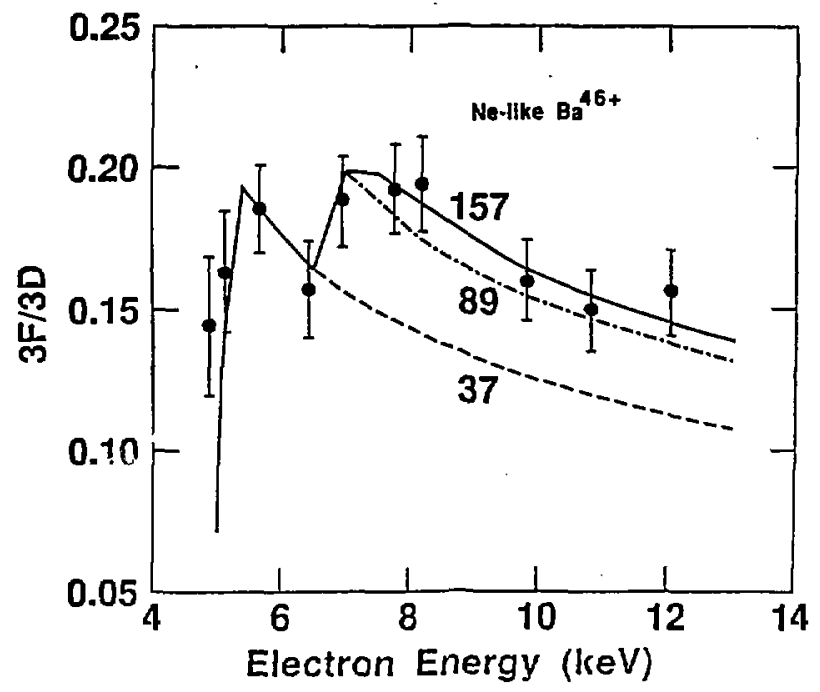

Fig. 9 - Messured ratio of the $3 \mathrm{~F}$ and $3 \mathrm{D}$ emission lines from Ne-like $\mathrm{Ba}^{46+}$. The curves show the results from niodels which include 37, 89, or 157 levels. 


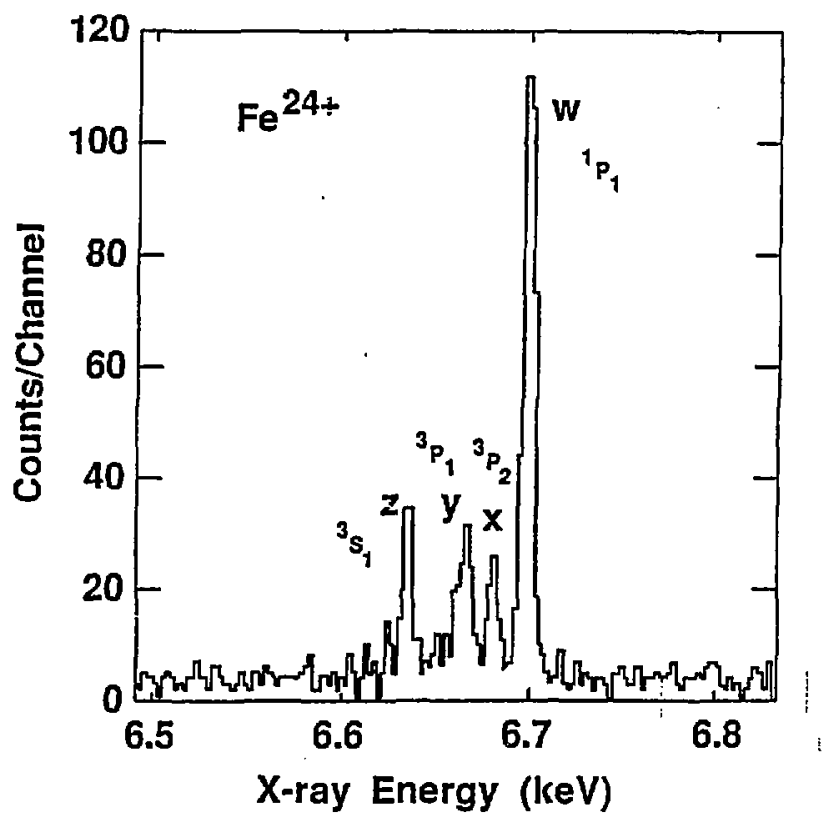

Fig. 10 - Spectrum of $n=2 \rightarrow 1$ transitions im He-like Fe ${ }^{24+}$ excited at an electron energy of $8.2 \mathrm{keV}$.

excited electrons in the $n=3,4,5$ shells. The comparison between the calculations and the data shows that cascade feeding from high-n levels is a significant population mechanism for neonlike ions, which cannot be ignored in theoretical models.

We are also studying excitation mechanisms in $H$-like and He-like systems. A typical $\mathrm{x}$-ray spectrum of the $\mathrm{n}=2 \rightarrow 1$ transitions in He-like iron is shown in Fig. 10. A series of such spectra, obtained at different electron energies, will be used to remove model dependent uncertainties which affect the use of these line ratios as a plasma diagnostic.

\section{SUMMARY}

The electron beam ion trap, with its capability for $\mathrm{x}$-ray spectroscopy, is now established as a unique tool for the study of highly charged ions. In this paper we have given examples of electron collision cross section measurements and precision transition-energy measurements performed with the EMIT at LLNL. The ionization stages available for $\mathrm{x}$-ray spectroscopy 
with EBIT already exceed those observed in the most advanced tokamaks, and essentially match the highest charge states studied in bearn-foil spectroscopy.

\section{ACKNOWLEDGMENTS}

Work performed under the auspices of the U.S. Department of Energy by the Lawrence Livermore National Laboratory under contract No. W-7405-ENG-48.

\section{REFERENCES}

1. R.E. Marrs, M.A. Levine, D.A. Knapp, and J.R. Henderson, Phys. Rey. Lett. 60, 1715 (1988).

2. M.A. Levine, R.E. Marrs, J.R. Henderson, D.A. Knapp, and M.B. Schneider, Phys. Ser. T22, 157 (1988).

3. R.E. Marrs, M.A. Levine, D.A. Knapp, and J.R. Henderson, in Electronic and Atomic Collisions, edited by H.B. Gilbody, W.R. Newell, F.H. Read, and A.C.H. Smith (North-Holland, Amsterdam, 1988), p. 209.

4. L. von Hamos, Ann. Phys. 17, 716 (1933).

5. P. Beiersdorfer et al. Phys. Rev A 34, 1297 (1986); P. Beiersdorfer et al., Phys. Rev. A 37, 4153 (1988).

6. G.A. Chandler, et al., Phys. Rev. A 39, 565, (1989); D.D. Dietrich, et al., Nucl. Inst. Meth. B 24/25, 301 (1987).

7. F. Bombarda, R. Giannella, E. Kallne, G.J. Tallents, F. Bely-Dubsu, P. Faucher, M. Cornille, J. Dubau, and A.H. Gabriel, Phys. Rev. A 37, 504 (1988).

8. H. Hsuan, M. Bitter, K.W. Hill, S. von Goeler, B. Grek, D. Johnson, L. C. Johnson, S. Sesnic, C.P. Bhalla, K.R. Karim, F. Bely-Dubau, and P. Faucher, Phys. R.ev. A 35, 4280 (1987).

9. K. Tanaka, T. Watanabe, K. Nishi, and K. Akita, Ap. J., 254 L59 (1982).

10. J.P. Briand, P. Charles, J. Ariarer, H. Laurent, C. Goldstein, J. Dubau, M. Loulergue, and F. Bely-Dubau, Phys. Rev. Lett. 52,617 (1984).

11. M.H. Chen, Phys. Rev. A 33, 994 (1986).

12. K.J. Reed, Phys. Rev. A 37, 1791 (1988).

13. M. Klapisch, private communications; A. Bar-Shalom, M. Klapisch, and J. Oreg, Phys. Rev. A 38, 1773 (1988). 